\title{
ALLOPURINOL IN THE THERAPY OF NEOPLASIA AND BLOOD DISEASES
}

\author{
METABOLIC ASPECTS \\ BY \\ R. W. E. WATTS* \\ London
}

The rate of nucleic acid turnover is increased in malignant tissue, and hyperuricaemia and hyperuricaciduria may occur when the condition is widespread as in the myeloproliferative disorders and reticuloses. Patients who are undergoing treatment which involves the dissolution of large amounts of malignant tissue are at risk from acute urinary obstruction. This may be either intrarenal, through the precipitation of uric acid crystals in the distal part of the nephron where the urine becomes concentrated and where acidification occurs (acute uric acid nephropathy), or postrenal, through calculi or loose aggregates of uric acid crystals.

This sudden increase in the rate of uric acid production occurs in the context of a complex clinical situation and the factors responsible for the development of urinary obstruction in some patients but not in others are obscure. The amount of nucleic acid breaking down and the rate at which this occurs are important, although in the case of leukaemia the pretreatment leucocyte count and the incidence of obstructive complications are not closely correlated. The rate of de novo purine biosynthesis may be important, either because the patient is also a congenital uric acid overproducer or because it has been accelerated by drug treatment as occurs when the 2-substituted thiadiazoles are given (Seegmiller, Grayzel, and Liddle, 1959; Krakoff and Balis, 1959; Seegmiller, Grayzel, Liddle, and Wyngaarden, 1963; Krakoff, 1965). The catabolism of large amounts of tissue protein superimposed on a state of chronic metabolic acidosis with hyperlacticacidaemia due to a high level of anaerobic metabolism in the tumour tissue (Field, Block, Levin, and Rall, 1966) could cause the excretion of a persistently acid urine and favour uric acid precipitation especially in a dehydrated patient. The complication may also be more

\footnotetext{
*The Medical Professorial Unit, St. Bartholomew's Hospital, London, E.C.1.
}

likely to occur if the neoplasia develops in subjects $\infty$ who always secrete a strongly acid urine (MetcalfeGibson, McCallum, Morrison, and Wrong, 1965) and are therefore genetically predisposed to form uric acid stones. Some of the drugs used as cytotoxic agents or to treat complications and symptoms of the primary disease are uricosuric, and so help to overload the renal tubular fluid with uric acid. Thus, both 6-azauridine and orotic acid (which is excreted when 6-azauridine is given) have uricosurio properties (Fallon, Frei, Block, and Seegmiller 1961), as have the adrenocorticosteroids (Ingbato Kass, Burnett, Relman, Burrows, and Sisson, 1951). large doses of salicylate (Yü and Gutman, 1959 and methicillin (Rieselbach, Bentzel, Cotlove, Frei, and Freireich, 1964).

The extent to which these factors can be influenced is limited, and until the introduction of allopurinol the only measure available for the prevention of the acute renal complications was the maintenance of a high rate of flow of alkaline urine. The possible presence of impacted uric acid calculi should also be excluded if anuria occurs during cytotoxic drug therapy.

Allopurinol inhibits xanthine oxidase and thereby reduces the oxidation of hypoxanthine to xanthine and of xanthine to uric acid. Thus, allopurinol treatment has the effect of sharing the purine load between uric acid, xanthine, and hypoxanthine. The solubilities of these compounds in urine are 15,5 , and $140 \mathrm{mg} / 100 \mathrm{ml}$. respectively at $p \mathrm{H} 5$, and 200,13 , and $150 \mathrm{mg} . / 100 \mathrm{ml}$. respectively at $p \mathrm{H} 7$ (Klinenberg, Goldfinger, and Seegmiller, 1965).

Comparisons between the total purine excretion in normal subjects and in patients with primary $N$ hyperuricaemia before and during allopurinol $\mathrm{N}^{\mathrm{N}}$ administration have usually shown diminution in the amount of total purine excreted during allopurinol administration. The reported magnitude of this 
effect has varied in different patients (Rundles, Wyngaarden, Hitchings, Elion, and Silberman, 1963; Klinenberg and others, 1965). McCollister, Gilbert, Ashton, and Wyngaarden (1964) showed that allopurinol ribonucleotide inhibits the enzyme which is responsible for the first stage of de novo purine biosynthesis, and it has been suggested that this is responsible for the reported discrepancy. It could, however, be due to increased re-utilization of hypoxanthine for adenine and guanine synthesis when xanthine oxidase is inhibited (Pomales, Bieber, Friedmann, and Hitchings, 1963).

The effects of allopurinol in vivo are apparent within 24 to 48 hours and reach their maximum in 3 to 5 days. The changes in the excretion of uric acid and the oxypurines (hypoxanthine plus xanthine) by a patient with acute lymphoblastic leukaemia who was given allopurinol prophylactically while in haematological remission and whose disease relapsed while he was taking the drug are shown in the Figure. The greatly increased purine load, which was excreted between about the 58th and the 70th day of the study, was shared between uric acid and the oxypurines, and the risk of acute uric nephropathy, which the patient had developed in the previous relapse, and with which he had pre- 3 sented on the first day of the study, was averted $\frac{D}{\infty}$. (Watts, Watkins, Matthias, and Gibbs, 1966). Other workers have also commented on the ease with which acute hyperuricaemia and hyperuric- $\overrightarrow{0}$ aciduria complicating the myeloproliferative diseases $\frac{}{\circ}$ and reticuloses can be averted by the use of allopuri- $\overline{\frac{\bar{m}}{}}$ nol (Krakoff and Meyer, 1965; Krakoff, 1965; De Conti and Calabresi, 1966; Vogler, Bain, Huguley, Palmer, and Lowrey, 1966).

Allopurinol slows the oxidation of 6-mercapto- $\overrightarrow{0}$ purine and azathiopurine (Elion, Callahan, Nathan, $\vec{\overrightarrow{ }}$ Bieber, Rundles, and Hitchings, 1963a), and Vogler and others (1966) report the case of a patient with acute leukaemia, who responded to a combination of allopurinol and 6-mercaptopurine having previously of been refractory to the latter drug. Xanthine oxidase is catalyses the oxidation of pteridins (Lowry, Bessey, and Crawford, 1949) and has a role in folic acid metabolism (Goodwin, 1963), but the influence of 9 allopurinol on the efficacy of the cytotoxic folic $v$ acid analogues requires further study.

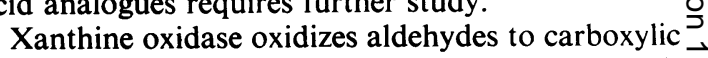
acids in vitro (Booth, 1938) and, although allopuri- $z$ nol inhibits the oxidation of glyoxylate to oxalate?

LEUCOCYTE COUNT

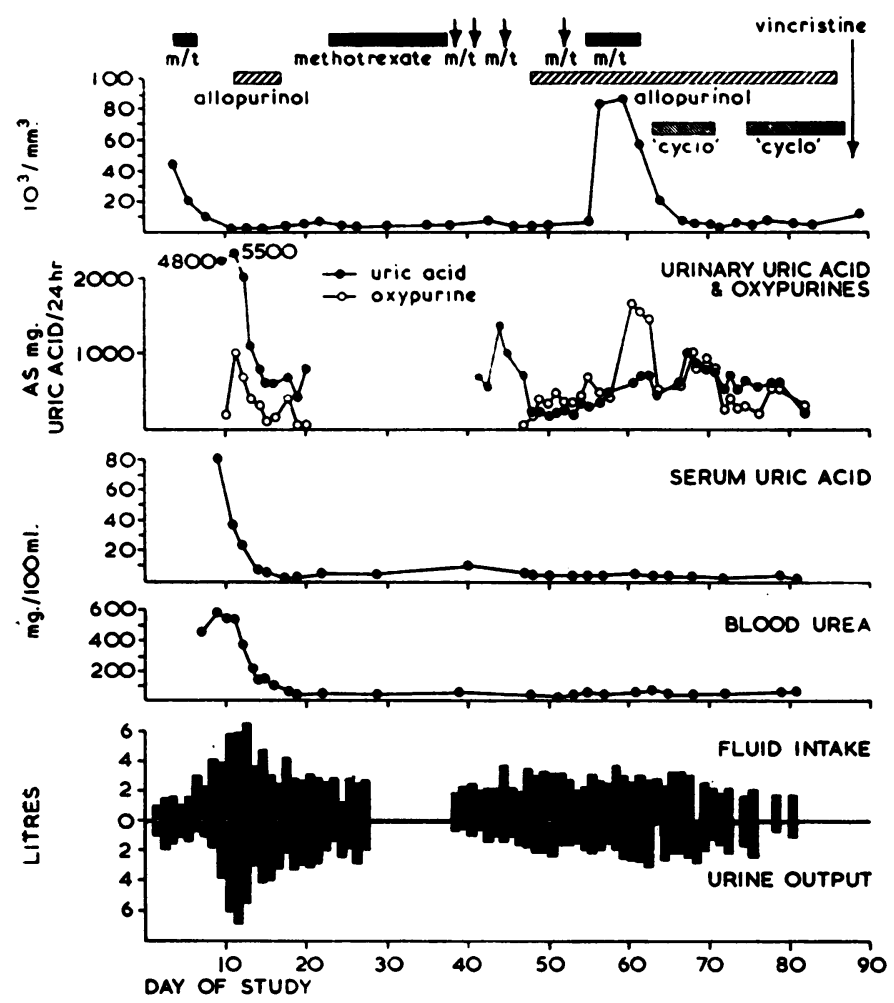

Fig. 1.-Haematological, biochemical, and fluidbalance data related to the patient with acutelymphoblastic leukaemia whose disease relapsed while he was being given allopurinol. $\mathbf{M} / \mathbf{t}=$ Methotrexate. Cyclo = Cyclophosphamide British Medical .Journal.] 
by the enzyme, it did not appreciably affect the glyoxylate oxidation to oxalate by human liver tissue in vitro or the oxalate excretion by two gouty patients (Watts and Gibbs, 1965; Gibbs and Watts, 1966). Thus, alternative metabolic pathways of aldehyde oxidation appear to be available when the enzyme is inhibited, and this would be important if other more toxic aldehydic metabolites have to be metabolized.

It is difficult to predict which patients will develop the acute obstructive urinary complications of cytotoxic therapy, but the following may be pointers to their likely occurrence and indications for giving allopurinol before and during cytotoxic and/or radiotherapy in addition to a high fluid intake and measures aimed at alkalinizing the urine:

(a) The presence of large amounts of leukaemic or other malignant tissue;

(b) A previous similar obstructive episode;

(c) Difficulty in maintaining an output of about 3 litres of alkaline urine per 24 hours;

(d) Urinary uric acid concentrations approaching $200 \mathrm{mg}$./100 ml. (the solubility of urate in urine at $p \mathrm{H} \mathrm{7}$ ) or marked hyperuricaemia (serum uric acid greater than $12 \mathrm{mg} . / 100 \mathrm{ml}$.) before definitive treatment is begun.

Allopurinol is also indicated in the management of patients with secondary gout or uric acid calculi associated with secondary hyperuricaemia. 